\title{
THREE-DIMENSIONAL EVALUATION OF SOFT TISSUE CHANGES FOLLOWING BONE-ANCHORED MAXILLARY PROTRACTION PROTOCOL IN PATIENTS WITH COMPLETE UNILATERAL CLEFT LIP AND PALATE
}

\author{
Eman Hossam Elabbassy*, Noha Ezzat Sabet**, Islam Tarek Hassan***, \\ Dina Hussein Elghoul ${ }^{* * * *}$ and Marwa Abdelwahab Elkassabby ${ }^{* * * * * *}$
}

\begin{abstract}
Objective: To evaluate three-dimensionally the soft tissue changes following maxillary protraction using bone-anchored maxillary protraction protocol (BAMP) in patients with complete unilateral cleft lip and palate (UCLP).

Material and methods: Twenty-eight growing patients (9-13 years old) with surgically repaired complete UCLP and skeletal Class III primarily due to maxillary deficiency were included in this study. After surgical placement of 4 custom-made modified surgical miniplates, patients were treated with BAMP protocol. Cone-beam computed tomography was taken after surgical placement of the miniplates (T1) and after 9 months (T2) to investigate treatment changes. Three-dimensional soft tissue measurements were assessed and correlated to the skeletal measurements.

Results: The results showed significant improvement in the soft tissue profile that closely followed the underlying skeletal changes. Forward movement of the upper lip was presented by increase in the linear measurements Ls-E line and Ss-ISP, increase in the H-angle and decrease in the nasolabial angle. No changes were found in the position of the lower lip or in the soft tissue chin. There was an increase in the angle of facial convexity. There was a statistically significant direct correlation between the amount of forward movement of the maxilla and the whole midface together with the forward movement of the upper lip.
\end{abstract}

Conclusions: BAMP protocol effectively improved the concave soft tissue profile in growing patients with UCLP.

KEYWORDS: Bone-anchored maxillary protraction, unilateral cleft lip and palate, concave soft tissue profile.

* Lecturer, Orthodontic Department, Faculty of Dentistry, Ain Shams University, Cairo-Egypt

** Professor, Orthodontic Department, Faculty of Dentistry, Ain Shams University Cairo, Egypt, Vice Dean of Post-Graduate Affairs, Faculty of Dentistry, Ain Shams University, Cairo, Egypt

*** Professor and Head of Department, Orthodontic Department, Faculty of Dentistry, Ain Shams University, Cairo, Egypt **** Associate Professor, Orthodontic Department, Faculty of Dentistry, Ain Shams University, Cairo- Egypt

****** Professor and Head of Department, Oral and Maxillofacial Surgery Department, Faculty of Dentistry, Ain Shams

University, Cairo, Egypt 


\section{INTRODUCTION}

Analysis of the soft tissue profile has recently emerged as the most important measure for assessing growth changes and treatment outcomes than the conventional skeletal analysis. ${ }^{1}$ It has the privilege of assessing the external appearance and so perhaps reflecting an outcome closer to that perceived by an observer.

Patients with unilateral cleft lip and palate (UCLP) are well known by their concave soft tissue profile resulting from the underlying Class III skeletal pattern. ${ }^{2}$ They usually suffer from a hypoplastic maxilla due to the stretching effect of the soft tissues resulting from early surgical lip closure. The primary goal for treatment of patients with CLP is to obtain an acceptable soft tissue profile that will be reflected on the psychological status of those debilitated patients.

Facemask therapy has been well known for its efficiency in correction of maxillary deficiency characteristic for patients with CLP. ${ }^{3}$ However, undesirable dental effects as well as skeletal changes have been reported. ${ }^{4-6}$ Although some of these side effects might be favorable for patients with CLP; like proclination of the maxillary incisors, and mesial movement with vertical eruption of the molars, yet excellent compliance with a somewhat burdensome extra-oral appliance is still required for 12 to 16 hours per day for 9 to 12 months for good clinical outcomes. ${ }^{7}$

Bone-anchored maxillary protraction protocol (BAMP) was introduced as an alternative for facemask therapy; modified miniplates are fixed to the maxilla and the mandible and the orthopedic force is applied by means of Class III intermaxillary elastics. This treatment approach produces pure skeletal effects without any dentoalveolar side effects and with minimal patient compliance which is limited to the replacement of the elastics and the maintenance of good oral hygiene..$^{8-10}$
Several studies investigated the dentoskeletal effects of this treatment approach which proved its efficiency to move the hypoplastic maxilla and the midface in patients with CLP forward, with no dentoalveolar side effects. ${ }^{8-10}$ However, its effect on the overlying soft tissues and whether the stretched upper lip that was previously surgically closed would respond to the forward movement of the underlying skeletal structures, has been investigated in limited two-dimensional (2D) studies. ${ }^{11}$ However, 2D studies have some inherent problems ranging from difficulty in landmark identification, to questions about the validity of assessing 3D structures in 2D projections. Furthermore, in patients with CLP, these problems are further complicated by the disruption of the normal anatomy of the maxilla, due to both the original defect and the surgical correction.

Thus, the aim of this study was to evaluate threedimensionally the soft tissue changes that followed the forward movement of the maxilla and the whole midface after treatment with BAMP protocol in patients with UCLP.

\section{MATERIALS AND METHODS}

This prospective study was conducted on 28 growing children with complete UCLP ranging in age from 9 to 13 years $($ mean $=10.8, \mathrm{SD}=1.2$ ). The subjects were selected from the outpatient clinic of Orthodontic Department and Cleft-CareCenter affiliated to Oral and Maxillofacial Surgery Department, Faculty of Dentistry, xxxx University. Patients were selected according to the following inclusion criteria: non-syndromic surgically repaired complete unilateral cleft of the lip, alveolar process and secondary palate, skeletal Class III primarily due to maxillary deficiency (determined by clinical examination including profile evaluation and confirmed by measuring SNA $<78^{\circ}$ ), pre-pubertal stage of skeletal maturity according to cervical vertebral maturation $(\mathrm{CVM})$ method $(\mathrm{CS} 1-\mathrm{CS} 3)^{12}$, mixed dentition (erupted lower permanent lateral 
incisors) or full permanent dentition stages, anterior cross-bite or edge-to-edge incisor relationship. None of the patients had previous orthopedic or orthodontic treatment and they were all medically free except for the CLP deformity.

Before start of treatment, informed consent and assent that were previously approved by the research ethics committee (REC) of Faculty of Dentistryxxxx University, were signed by the parents and the patients respectively.

After pre-surgical preparation, the surgical procedure was performed under general anesthesia. For the maxillary arch, a horizontal vestibular incision was cut down to the level of bone above the mucogingival junction bilaterally at the site of insertion of the maxillary miniplates; between the first permanent molar and the second premolar or the second primary molar. In the mandibular arch, a median vestibular horizontal incision was cut down to the level of bone exposing the whole area from canine to canine below the mucogingival junction. Four custom-made modified surgical titanium miniplates (Modern Techniques Center, Cairo, Egypt) that were accepted by the REC of Faculty of Dentistry- xxxx University, were manufactured for this research from the biocompatible titanium alloy Grade 2. For every patient, one miniplate was placed in each infrazygomatic buttress of the maxilla and one in the anterior mandible on each side; between and inferior to the right and left permanent lateral incisor and canine. The modified miniplates were secured to bone by 3 maxillary (diameter: $2.3 \mathrm{~mm}$, length: $6 \mathrm{~mm}$ ) and 2 mandibular (diameter: $2.3 \mathrm{~mm}$, length: $5 \mathrm{~mm}$ ) monocortical self-drilling titanium miniscrews. The attachment hook of each miniplate perforated the attached tissue at or near the mucogingival junction. All mucoperiosteal flaps were sutured by $4 / 0$ resorbable sutures. This was followed by the placement of small plastic sleeves around the head of each miniplate to avoid soft tissue overgrowth around them during the healing period. Post-operative medications were prescribed for every patient; antibiotics and NSAIDs for 5 days. Soft diet and oral hygiene instructions were given with strong emphasis on brushing around the miniplates with a soft toothbrush and continuous use of antiseptic mouth wash 3 times daily. Patients were recalled 1 week after the surgical procedure to ensure adequate soft tissue healing.

The miniplates were loaded to start the orthopedic protraction protocol 3 weeks after surgery using Class III intermaxillary elastics which were chosen individually for each case to provide an initial force of approximately $150 \mathrm{gm} / \mathrm{side}$, increased to $200 \mathrm{gm}$ after 1 month of traction and to $250 \mathrm{gm}$ after 2 months. ${ }^{8}$ One elastic was placed on each side to give vectors of force downward and forward for the maxilla, and backward and upward for the mandible. The force level was measured in maximum intercuspation by means of a force tension gauge (Correx, Koeniz, Switzerland). The patients were instructed to wear the elastics 24 hours per day and to replace them once a day and whenever a loss of elastic was experienced. An evaluation chart was given to every patient to record the duration of elastics' wear every day. For all patients, after 2 to 3 months of intermaxillary traction, a removable acrylic posterior bite-plane was placed in the mandibular arch to eliminate occlusal interference in the incisor region (Figure 1). Patients were instructed to wear it full time except during meals.

In patients where mobility of any of the 4 inserted miniplates was detected, the patient was instructed to discontinue the elastics for few days with emphasis on brushing and washing around the head of the involved miniplates. The protraction treatment was then re-continued after the stability was improved.

Three-dimensional assessment of the applied protocol was done using cone beam computed tomography (CBCT) scans that were taken for every patient 1 week after placement of the miniplates (T1) 
and after 9 months (T2). All images were acquired using $i$-CAT ${ }^{\circledR}$ scanner Next Generation (Model 17/19 series; Imaging Sciences International, Hatfield, PA). Upon scan completion, the projection data were reconstructed with i-CAT software to create a data set with a voxel size of $0.125 \mathrm{~mm}$ in $\mathrm{X}, \mathrm{Y}$ and $\mathrm{Z}$ directions. The reconstructed data were saved in digital imaging and communications in medicine (DICOM) file format. The DICOM files were then downloaded and reconstructed into volumetric images using InVivo 5 software (version 5.2; Anatomage, San Jose, CA, USA). Because the studied sample was composed of growing children, a customized 3D craniofacial analysis was developed for this study to exclude the growth factor and to be sure that any improvement in the measurements taken, were only due to the applied treatment modality and not due to normal growth and development. The reference planes used in this analysis depend on stable cranial base structures that do not undergrow remodeling after the age of 4 to $5 .{ }^{13,14}$ The analysis consisted of the following:

1- A new coordinate reference system including:

a- Modified midsagittal plane (MSP): connecting between points $(\mathrm{T}){ }^{15}$, point ELSA ${ }^{16}$ and Basion (Ba).

b- Interspinosal plane (ISP): connecting right and left foramen spinosum perpendicular to the modified MSP.

c- T perpendicular plane (TPP): drawn from point $(\mathrm{T})$ perpendicular to the modified MSP and ISP.

2- Skeletal measurements (A-ISP, Or-ISP, KR-ISP, B-ISP, Pog-ISP). (Figure 2).

3- Soft tissue measurements (Ls-E line, Ss-ISP, Li-E line, Si-ISP, Pog'-ISP, G'-Sn'-Pog', H-angle, Col-Sn'-Ls) (Figure 3).

To assess the reliability of the measurements, 8

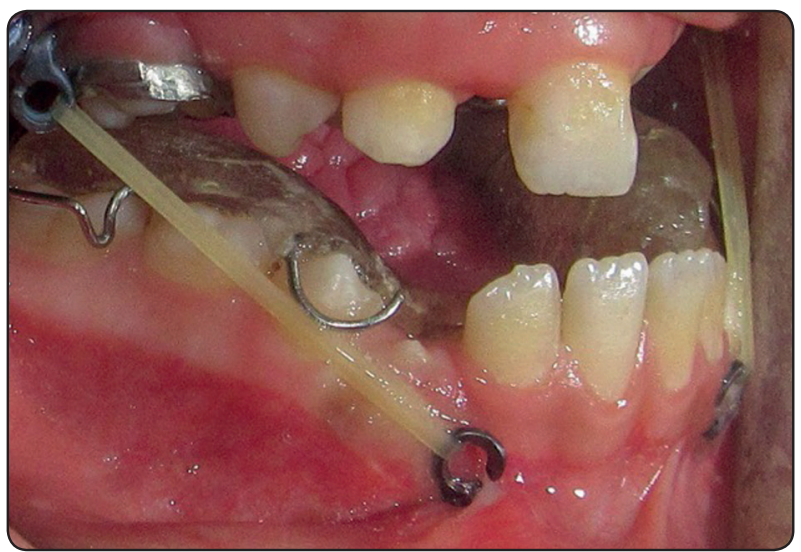

Fig. (1): Class III intermaxillary elastics applying orthopedic protraction force with a removable bite-plane in the mandibular arch.

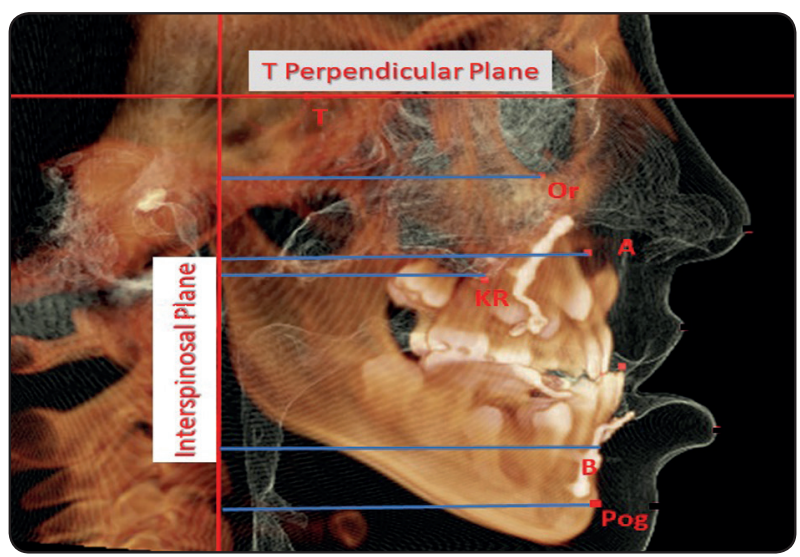

Fig. (2): 3D Skeletal measurements

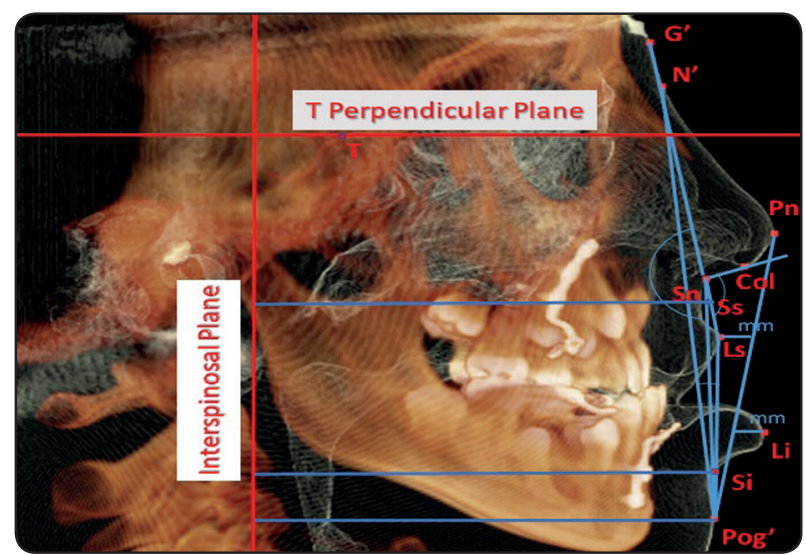

Fig. (3): 3D Soft tissue measurements 
1 week after the initial measurement by the same investigator and by another investigator. Cronbach's alpha reliability coefficient results showed very good intra-observer and inter-observer agreement with Cronbach's alpha value not less than 0.800 for all the variables.

\section{Statistical analysis}

The collected data were tabulated then statistically analyzed utilizing SPSS software (version 20.0; IBM, Armonk NY). Numerical data were explored for normality by checking the data distribution and using Kolmogorov-Smirnov and Shapiro-Wilk tests for further choice of appropriate parametric and non-parametric tests. Descriptive statistics were computed for all the variables. The significance level was set at $P \leq 0.05$. Most of the data showed parametric distribution where paired t-test was used to test the significant differences between $\mathrm{T} 1$ and $\mathrm{T} 2$ measurements. For non-parametric data (Li-E line (mm), Gn'-Sn-Pog' ') Wilcoxon signed-rank test was used. Spearman's correlation coefficient was used to determine the correlation between different quantitative variables.

\section{RESULTS}

The changes between $\mathrm{T} 1$ and $\mathrm{T} 2$ are shown in Table 1 and Figure 4, which showed significant active treatment effects. There was significant forward movement of the maxilla represented by increased distance between point $\mathrm{A}$ and ISP $(3 \mathrm{~mm})$. Points Orbitale and key ridge, representing the midface, were significantly displaced forward.

TABLE (1). Mean, standard deviation (SD) values and results of paired t-test and Wilcoxon signed rank test for the changes after treatment

\begin{tabular}{|c|c|c|c|c|c|c|c|}
\hline \multirow{2}{*}{ Parameter } & \multicolumn{2}{|c|}{ Pre-treatment (T1) } & \multicolumn{2}{|c|}{ Post-treatment (T2) } & \multicolumn{2}{|c|}{ Change } & \multirow{2}{*}{ P-value } \\
\hline & Mean & SD & Mean & SD & Mean & SD & \\
\hline \multicolumn{8}{|c|}{ Skeletal measurements } \\
\hline A-ISP (mm) & 62.14 & 2.52 & 65.31 & 2.70 & 3.17 & 1.37 & $0.007 *$ \\
\hline Or-ISP (mm) & 51.01 & 3.35 & 53.78 & 3.61 & 2.77 & 1.55 & $0.016 *$ \\
\hline KR-ISP (mm) & 36.76 & 2.35 & 38.48 & 2.47 & 1.72 & 1.05 & $0.021 *$ \\
\hline $\mathrm{B}-\mathrm{ISP}(\mathrm{mm})$ & 67.77 & 1.52 & 67.58 & 1.13 & -0.19 & 0.42 & 0.374 \\
\hline Pog-ISP (mm) & 69.65 & 1.99 & 70.44 & 2.90 & 0.79 & 1.13 & 0.195 \\
\hline \multicolumn{8}{|c|}{ Soft tissue measurements } \\
\hline Ls-E line (mm) & -7.49 & 1.33 & -4.84 & 0.76 & 2.65 & 1.43 & $0.043 *$ \\
\hline Ss-ISP (mm) & 75.40 & 1.80 & 78.1 & 1.36 & 2.70 & 1.37 & $0.012 *$ \\
\hline Li-E line (mm) & 0.49 & 2.36 & 0.62 & 2.06 & 0.13 & 0.47 & 0.500 \\
\hline Si-ISP (mm) & 78.25 & 2.08 & 78.69 & 2.42 & 0.44 & 0.71 & 0.239 \\
\hline Pog'-ISP (mm) & 80.62 & 1.39 & 81.69 & 1.99 & 1.07 & 1.32 & 0.143 \\
\hline G'-Sn'-Pog, ${ }^{\circ}$ & -1.44 & 3.09 & 1.76 & 2.99 & 3.20 & 1.75 & $0.042 *$ \\
\hline $\mathrm{H}$-angle ${ }^{\circ}$ & 6.22 & 1.4 & 8.12 & 0.8 & 1.90 & 1.34 & $0.034 *$ \\
\hline Col-Sn'-Ls ${ }^{\circ}$ & 100.37 & 3.06 & 98.14 & 3.01 & -2.23 & 1.31 & $0.019 *$ \\
\hline
\end{tabular}

*, Significant at $P \leq 0.05$ 


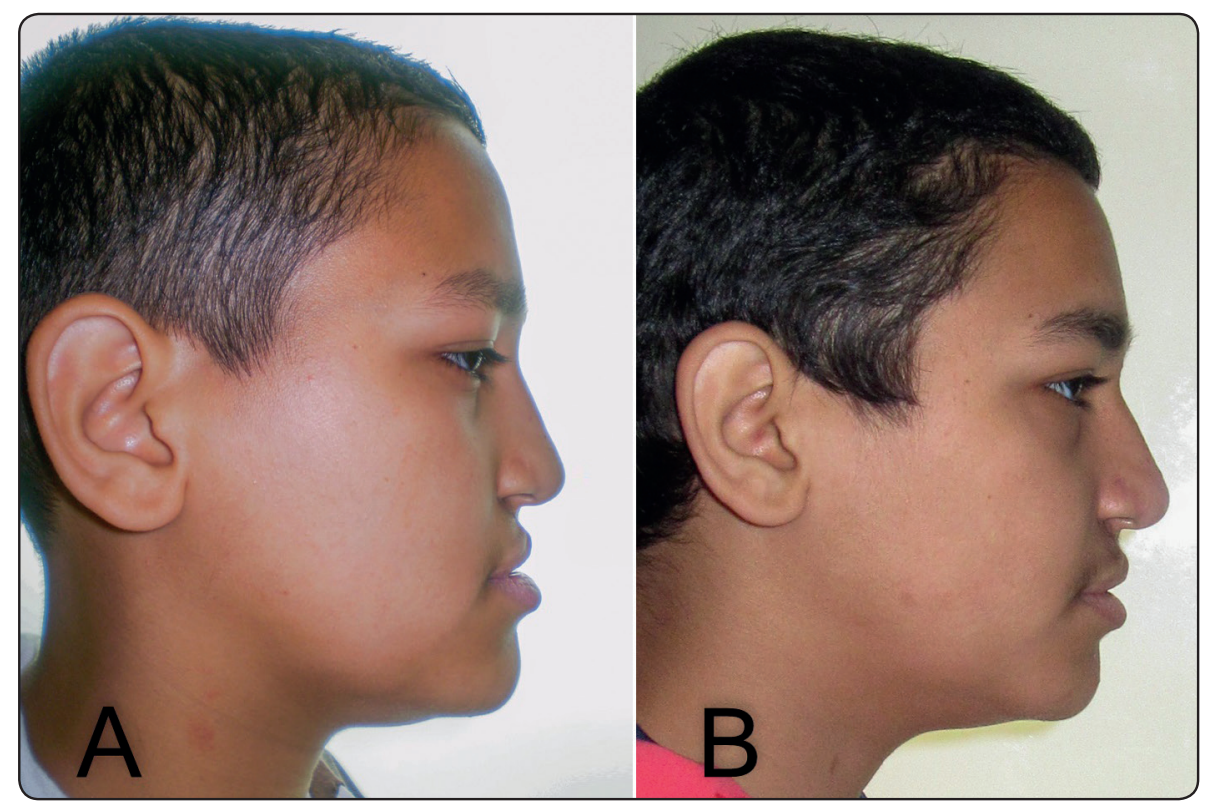

Fig. (4) Extra-oral (profile) view for a patient treated with BAMP protocol: A, before and $\mathrm{B}$, after 9 months treatment.

The mandibular growth was restrained. There was significant improvement in the soft tissue profile represented by forward movement of the upper lip with no change in the lower lip position or the soft tissue chin, increase in the soft tissue convexity, increase in the $\mathrm{H}$-angle and a decrease in the nasolabial angle. There was a statistically significant direct (positive) correlation between A-ISP (mm) and Ls-E line (mm), Or-ISP (mm) and Ls-E line (mm) as well as KR-ISP (mm) and Ls-E line (mm) as shown in Table 2.

TABLE (2). Results of Spearman's correlation coefficient for the correlation between changes in different measurements

\begin{tabular}{ccc}
\hline Measurements & $\begin{array}{c}\text { Correlation } \\
\text { coefficient }\end{array}$ & P-value \\
\hline A-ISP (mm) and Ls-E line (mm) & 0.981 & $\mathbf{< 0 . 0 0 1 *}$ \\
\hline Or-ISP (mm) and Ls-E line (mm) & 0.960 & $<\mathbf{0 . 0 0 1 *}$ \\
\hline KR-ISP (mm) and Ls-E line (mm) & 0.939 & $\mathbf{< 0 . 0 0 1 *}$ \\
\hline
\end{tabular}

*, Significant at $P \leq 0.05$

\section{DISCUSSION}

Three-dimensional maxillary collapse together with a concave soft tissue profile, is a typical feature for patients with CLP. ${ }^{17}$ Their treatment will eventually require maxillary advancement to correct maxillary hypoplasia and improve the facial esthetics. ${ }^{18}$ This could be done by growth modification at an early age, distraction osteogenesis that can be carried as early as 5 years of age, later in adolescence or even during adulthood or orthognathic surgery when growth ceases..$^{19}$ Growth modification at an early age is important for patients with CLP even if full correction of the skeletal problem will not be achieved. Improving the facial esthetics in the pre-adolescent and adolescent stages instead of postponing the surgical treatment until completion of growth may have a favorable effect on the self-esteem and the psychological development of those debilitated patients during their puberty. Moreover, the magnitude of surgery needed after early intervention would be expected to be less than in patients who had no growth modification. ${ }^{20}$

BAMP protocol became one of the most favourable treatment modalities for growth 
modification in patients with CLP. ${ }^{10,11,21,22}$ It was found to be capable of moving the hypoplastic maxilla and the whole midface forward even in the presence of the streching effect of the post-surgical scarring found in those patients. Furthermore, it was found to restraint the mandibular growth without any dentoalveolar side effects and with minimal patient compliance.

Although the aim of this study was to assess the soft tissue changes resulting from applying the BAMP protocol in patients with CLP, however, some skeletal measurements that were published in a previous study were mentioned here. ${ }^{10}$ The changes in the underlying supporting bony bases can explain the soft tissue changes that were found out in this study. Forward movement of the maxilla was indicated by the increase in the linear measurement between ISP representing the vertical reference plane and point A representing the anterior limit of the maxilla (Table 1). Another interesting finding was the forward movement of both the infraorbital and key ridges that was obvious both clinically and statistically.

The improvement in the upper lip projection, indicated by the increase in the linear measurements of the Ls to the E Line and Ss to the vertical reference plane (ISP), the increase in the $\mathrm{H}$-angle and by the decrease in the nasolabial angle, is a direct reflection to the forward movement of the maxilla and the whole midface induced by the applied treatment protocol which agrees with the previous studies applying the BAMP protocol. ${ }^{11,23,24}$

As this treatment protocol was able to restraint the mandibular growth without producing any change in the mandibular measurements, the lower lip did not show change in their position which is a favorable result in patients with CLP.

The clinical improvement of the soft tissue profile of the treated patients was confirmed as well by the statistical significant improvement in the angle of facial convexity that was consistent with the studies carried by De Clerck et al. ${ }^{23}$ and Jahanbin.${ }^{11}$

The high degree of correlation between the amount of forward maxillary movement, forward movement of the infra-orbital and key ridges, together with the forward movement of the upper lip relieved the doubts about the response of the surgically manipulated upper lip to the movement of the underlying skeletal bases. This finding ensures that the upper lip can move forward proportionally to the amount of forward movement of the maxilla and the midface under treatment with BAMP protocol, and hence improving the soft tissue profile and facial esthetics in patients with UCLP.

\section{Limitations}

The study showed that BAMP protocol is an effective treatment for the correction of the upper lip position together with improving the facial esthetics in patients with CLP. However, this study has some limitations; the variation in the timing and technique of early surgical lip and palatal closure might produce variable amount of scarring and stretching, that produces variable resistance to the protraction force among different patients and consequently different amounts of facial esthetics improvement, which needs to be standardized.

\section{CONCLUSIONS}

BAMP protocol effectively improved the concave soft tissue profile in growing patients with UCLP. It was capable of moving the maxilla, the whole midface and the overlying upper lip forward while maintaining the lower lip in its position.

\section{REFERENCES}

1- Bearn DR, Sandy JR, Shaw WC. Cephalometric soft tissue profile in unilateral cleft lip and palate patients. Euro J Orthod. 2002;24:277-284.

2- Coccaro PJ, Pruzansky S. Longitudinal study of skeletal and soft tissue profile in children with unilateral cleft lip and palate. Cleft Palate J. 1965;2:1-12. 
3- Ahn HW, Kim KW, Yang IH, Choi JY, Baek SH. Comparison of the effects of maxillary protraction using facemask and miniplate anchorage between unilateral and bilateral cleft lip and palate patients. Angle Orthod. 2012;82:935941.

4- Zhou YH, Ding P, Lin Y. Facemask therapy with miniplate implant anchorage in a patient with maxillary hypoplasia. Chin Med J (Engl). 2007;120:1372-1375.

5- Lee NK, Yang IH, Baek SH. The -term treatment effect of facemask therapy in Class III patients based on the anchorage device: miniplates vs rapid maxillary expansion. Angle Orthod. 2012;82:846-852.

6- Morales-Fernandez M, Iglesias-Linares A, Yanez-Vico RM. Bone- and dentoalveolar anchored dentofacial orthopedics for Class III malocclusion: New approaches, similar objectives? A systematic review. Angle Orthod. 2013;83:540-552.

7- Heymann GC, Cevidanes LH, Conelis MA, De Clerck HJ, Tulloch CJ. Three-dimensional analysis of maxillary protraction with intermaxillary elastics to miniplates. Am J Orthod Dentofacial Orthop. 2010;137:274-284.

8- De Clerck H, Cevidanes L, Baccetti T. Dentofacial effects of bone-anchored maxillary protraction: A controlled study of consecutively treated Class III patients. Am J Orthod Dentofacial Orthop. 2010;138:577-581.

9- Nguyen T, Cevidanes L, Cornelis MA, Heymann G, De Paula LK, De Clerck H. Three-dimensional assessment of maxillary changes associated with bone anchored maxillary protraction. Am J Orthod Dentofacial Orthop. 2011;140:790-798.

10- Elabbassy EH, Sabet NE, Hassan IT, Elghoul DH, Elkassaby MA. Bone-anchored maxillary protraction in patients with unilateral cleft lip and palate: Is maxillary expansion mandatory? Angle Orthod. 2020;90:539-547.

11- Jahanbin A, Kazemian M, Eslami N, Pouya IS. Maxillary protraction with intermaxillary elastics to miniplates versus bone-anchored facemask therapy in cleft lip and palate patients. J Craniofac. 2016;27:1247-1252.

12- Baccetti T, Franchi L, McNamara JA Jr. The cervical vertebral maturation (CVM) method for the assessment of optimal treatment timing in dentofacial orthopedics. Semin Orthod. 2005;11:119-129.

13- Waitzman AA, Posnick JC, Armstrong DC, Pron GE. Craniofacial skeletal measurements based on computed
tomography:Part II. Normal values and growth trends. Cleft Palate Craniofac J. 1992;29:118-128.

14- Sgouros S, Natarajan K, Hockley AD, Goldin JH and Wake M. Skull base growth in childhood. Pediatr Neurosurg. 1999;31:259-268.

15- Viazis AD. The cranial base triangle. J Clin Orthod. 1991;25:565-570.

16- Lagravere MO, Major PW. Proposed reference point for 3-dimensional cephalometric analysis with cone-beam computed tomograpghy. Am J Orthod Dentofacial Orthop. 2005;128:657-660.

17- Graber TM. A cephalometric analysis of the developmental pattern and facial morphology in cleft palate. Angle Orthod. 1949; 19:91-100.

18- Ross RB. Treatment variables affecting facial growth in complete unilateral cleft lip and palate: Part 7. An overview of treatment and facial growth. Cleft Palate J. 1987;24:71-77.

19- Figueroa AA, Polley JW. Management of severe cleft maxillary deficiency with distraction osteogenesis: Procedure and results. Am J Orthod Dentofacial Orthop. 1999;115:112.

20- De Clerck HJ, Proffit WR. Growth modification of the face: A current perspective with emphasis on Class III treatment. Am J Orthod Dentofacial Orthop. 2015;148:3746.

21- Yatabe M, Garib DG, Faco RA, De Clerck H, Janson G, Nguyen T, Cevidanes LHS, Ruellas AC. Bone-anchored maxillary protraction therapy in patients with unilateral cleft lip and palate: 3-dimensional assessment of maxillary effects. Am J Orthod Dentofacial Orthop. 2017;152:327335 .

22- Ren Y, Steegman R, Dieters R, Jansma J, Stamatakis H. Bone-anchored maxillary protraction in patients with unilateral complete cleft lip and palate and Class III malocclusion. Clin Oral Inves. 2019;23:2429-2441.

23- De Clerck HJ, Cornelis MA, Cevidanes LH, Heymann GC, Tulloch CJ. Orthopedic traction of the maxilla with miniplates: A new perspective for treatment of midface deficiency. J Oral Maxillofac Surg. 2009;67(10): 2123-2129.

24- Elnagar MH, Elshourbagy E, Ghobashy S, Khedr M, Evans CA. Comparative evaluation of 2 skeletally anchored maxillary protraction protocols. Am J Orthod Dentofacial Orthop. 2016;150:751-762. 